\title{
A Proficient Bee Colony-Clustering Protocol to Prolong Lifetime of Wireless Sensor Networks
}

\author{
Aruna Pathak (iD) \\ Dept of Electronics and Communication Engineering, Government Engineering College Bharatpur, Rajasthan, India \\ Correspondence should be addressed to Aruna Pathak; aruna.pathak@gmail.com
}

Received 17 August 2018; Revised 14 November 2018; Accepted 23 January 2020; Published 25 February 2020

Academic Editor: Youyun Xu

Copyright (c) 2020 Aruna Pathak. This is an open access article distributed under the Creative Commons Attribution License, which permits unrestricted use, distribution, and reproduction in any medium, provided the original work is properly cited.

In wireless sensor network, replacement of node's battery is very tough task in hostile environments. Therefore, to maximize network lifetime is the ultimate solution. Dividing the sensing region of wireless sensor network into clusters is an excellent approach to gain high energy efficiency and to enhance lifetime of the network. On the other hand, heads of the cluster need additional energy because of additional work such as obtaining data from its member nodes, aggregation of their data, and finally sending it to the base station. To enhance the lifetime of these networks, proper selection of heads plays a vital role. In this paper, we propose proficient bee colony-clustering protocol (PBC-CP) which is based on artificial bee colony algorithm. In PBC-CP approach, we have taken important factors for selection of heads such as node's energy, degree of node, and distance from base station to node. For transmitting the data from cluster head to base station, it chooses the energy-efficient path which further minimizes the energy consumption of sensor network. Simulation experiments show the effectiveness of our proposed approach.

\section{Introduction}

Wireless sensor network (WSN) contains a huge number of tiny sensor nodes. These tiny nodes have sensing, computation, and wireless communications capabilities [1]. The WSN model is shown in Figure 1. The sensing area is the area where sensor nodes are deployed either $t$ randomly or installed manually. They gather the information from the sensing area, process it, and send wirelessly either to other nodes or to an external base station (BS). BS is a centralized point of control within the network. It may be a stationary or a movable node. BS is joined to an accessible communications infrastructure or to the Internet.

WSNs have found applications in business, home, medical, transport, industrial production, real-time control, defence, emergency, disaster relief management, etc. They are also used in supervising of remote or inaccessible environment applications $[2,3]$. It is a very tedious job to replace or even refill the attached battery of the node in many applications, especially supervising the hostile environment. The limited energy source is the main restriction of these networks $[4,5]$. The challenge of prolonging lifetime of the network has led to an increased research interest from the scientific community. As a result, researchers have proposed many techniques like duty cycling, data reduction, and topology management for enhancing the network's lifetime. Node's energy can be saved with duty cycling strategy that permits sensor nodes to go to sleep when they are not in use [6-10]. The data reduction method also reduces the energy consumption with the help of minimizing the quantity of information generated, processed, and transmitted [11-13]. The topology management saves the energy consumption of nodes by constructing and preserving a reduced set of nodes [14-16]. Cluster-based routing techniques seem to be most suitable for prolonging the lifetime of WSNs [17-19]. In clustering, the entire region is segregated into various groups termed as clusters and all groups are led by a particular head called as cluster head $(\mathrm{CH})$. These leaders are accountable for gathering the information from its member nodes inside the clusters, then aggregate the recieved data and send it to BS by single-hop trasmission model or multi-hop trasmission model. In the single-hop trasmission model, $\mathrm{CH}$ sends data diresctly to $\mathrm{BS}$. In the multi-hop trasmission model, $\mathrm{CH}$ uses some intermediate nodes, i.e., other $\mathrm{CHs}$, to forward the data towards BS. Selection of the heads is an extremely vital task for enhancing the lifetime of network. Appropriate selection 


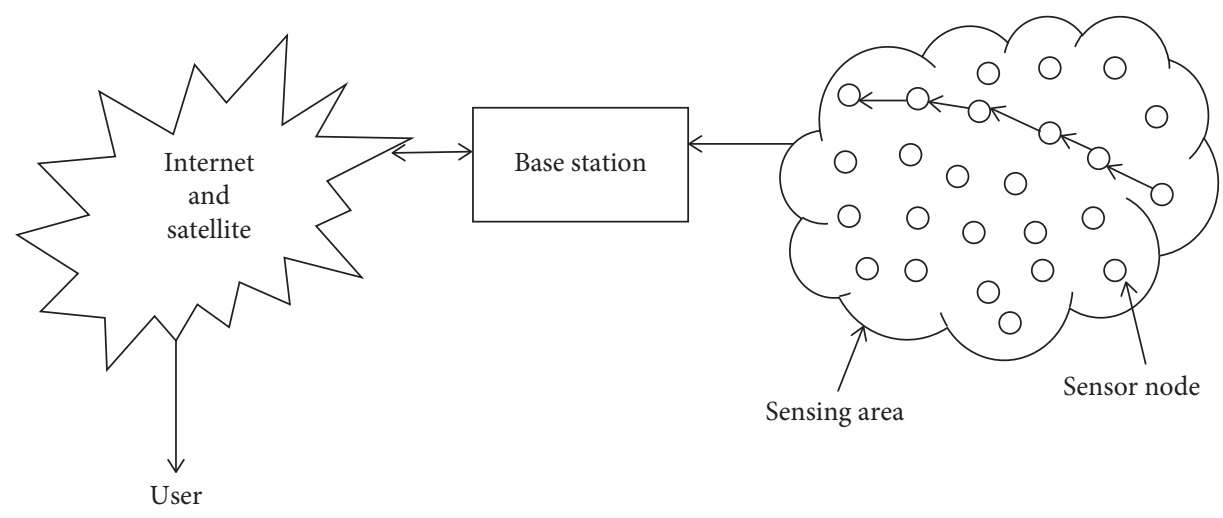

FIgURE 1: Wireless sensor network model.

has a number of impacts on the energy conservation of member nodes as well as effect on the routing method.

Low energy adaptive clustering hierarchy (LEACH) is a recognized clustering algorithm in WSNs [20]. CHs are selected on probability basis and rotated in each round for achieving energy balance. This protocol could gain partial success because it is entirely distributed protocol. In distributed protocol, more energy is required to transmit the packet. Clustering protocols have been also implemented with fuzzy logic [21-25]. Swarm intelligence offers proficient metaheuristic tools that can be efficiently applied in WSNs. Clustering is a well-known optimization problem. The swarm intelligence is efficiently solving this issue as surveyed in [26-30]. Ant colony optimization metaheuristic has also been applied in clustering [31]. Particle swarm optimization (PSO) algorithm is also used in clustering optimization. The protocol presented in [32] utilizes PSO for cluster head selection taking residual energy, intracluster distance, and node degree as fitness function. A hybrid centralized protocol combining harmony search algorithm (HSA) and PSO is also used for clustering in sensor networks [33]. Bee colony metaheuristic achieved success in resolving the clustering problem in WSNs [34-38].

In this paper, a proficient bee colony-clustering protocol (PBC-CP) is presented. Selection of heads of cluster is an optimization problem that is NP-hard in nature. Artificial bee colony (ABC) algorithm is a proficient nature inspired algorithm that can be a good option for such NP-hard problem because of its easiness of implementation and high quality of solution. The proposed protocol gains the best result with the appropriate selection of head of cluster on the basis of node's energy, degree of node, and distance from BS to node. The energy-efficient transmission of data from node to the base station further enhances its performance. The multihop transmission of data between adjacent $\mathrm{CHs}$ is followed on the basis of residual energy of nodes rather than direct transmission from $\mathrm{CH}$ to $\mathrm{BS}$. The major contributions of the paper are as follows:

(i) Firstly, the bee colony model is presented considering its applicability in clustering WSNs.

(ii) Secondly, proficient bee colony-clustering protocol is proposed focusing on phasewise description, functional pseudocode steps, and network model. (iii) Thirdly, the proposed clustering protocol is comparatively evaluated considering various network performance metrics.

The rest of the paper is organized as follows. Related work is described in Section 2. In Section 3, the details of the bee colony model and PBC-CP are presented. Section 4 discusses the experimental setup and performance evaluation of the proposed PBC-CP. Conclusion and future scope are presented in Section 5.

\section{Related Work}

Energy conservation is essential to prolong the whole network's lifetime. Network lifetime can be defined as the time elapsed until the first node in the network depletes its energy $[39,40]$. Cluster-based routing techniques seem to be most suitable for prolonging the lifetime of WSNs [17-19]. These methods also bring down energy consumption within a cluster by performing data aggregation and fusion. Low energy adaptive clustering hierarchy (LEACH) protocol [20] is a recognized clustering algorithm. However, there are certain drawbacks of this protocol. Some of them are as follows:

(1) It selects $\mathrm{CHs}$ based on probability which leads to two adverse consequences. First, there is a load imbalance among the $\mathrm{CH}$ due to nonassurance of uniform distribution of $\mathrm{CH}$ in the network. Second, low energy node may be chosen as $\mathrm{CH}$ s wich is not capable enough to do additional work of heads such as fusing the data obtained from its members and transfer this fused data to the BS.

(2) The CHs send their data to the BS in one hop transmission. They bear the energy expenditure of long range transmission. The $\mathrm{CH}$ that is distant from the BS diminishes its energy faster than the other $\mathrm{CHs}$ in the network, which are not so distant.

(3) In each round, the protocol has to do the process for selecting the new $\mathrm{CHs}$ and forming new clusters. This further increases the operating cost of the set up phase.

The authors in [41] tried to solve the problem of nonuniform load distribution of cluster heads. However, 
the scheme presented in [41] needs a node positioning system such as GPS that causes the system to be costly. In addition, GPS necessitates supplementary energy consumption; hence, it requires larger size hardware. The authors in [42] presented a new idea based on thresholds for sending node's data. However, it is difficult to calculate the precise value of these thresholds because this protocol is not appropriate for monitoring applications where data are continuously reported to the BS. In the paper presented in [43], efficient clustering scheme is presented where the $\mathrm{CH}$ nominees struggle to be promoted as heads. If a node could not find another node with more residual energy than itself, it takes up the responsibility of the head. This algorithm forms clusters of varying sizes using distance from the BS as metric. Hybrid energy-efficient distributed clustering [44] decides $\mathrm{CH}$ based on two important parameters: residual energy of every node and intracluster communication cost. Energy-efficient hierarchical clustering protocol [45] partitions the network into hierarchy of layers. Lowest level $\mathrm{CHs}$ collect information from members and aggregate it. The aggregated information from the lowest layer is then sent to the heads of the subsequent layer. This method repeats itself recursively until all the data have reached the BS. Stable election protocol [46] highlights the impact of heterogeneity of nodes regarding the energy of the nodes. In clustering algorithm via waiting timer [47], protocol node degree is taken into consideration for the selection of cluster heads. Autonomous clustering via directional antenna [48] algorithm uses directional antennas to decrease the redundancy in sensing the data in sensor networks. Two-level LEACH [49] protocol has two types of CHs, namely, primary heads and secondary heads. Network is divided into outer and inner layers. Primary heads are accountable for aggregating the data in outer layer, and the secondary heads are responsible for inner layer. LEACH with distance-based thresholds [50] algorithm selects CHs with modified probability. This approach optimally balances consumption of energy among the nodes. In [51], the parameter for the selection of head is dependent on the neighbours like distance between the nodes and the number of its neighbouring nodes within communication reach. The main focus of Ying et al.'s algorithm [52] is to balance the load with uniform and nonuniform node distribution in the network. The link aware clustering method (LCM) [53] initiates a new function, called predicted transmission count (PTC), to calculate the nominee conditions. The position of the nodes, transmitted power, residual energy, and link quality are used as the parameters to derive the PTC. The PTC demonstrates the potential of an applicant for persistent transmissions to any specific neighbouring node. In energy-efficient LEACH (EELEACH) [54] protocol, the criteria of selection of the head is based on the function of spatial density. The protocol considers the Gaussian distribution model for deployment of sensors. Hence, it is not suited to the applications where sensor nodes cannot be deployed manually.

Fuzzy logic-dependent clustering protocol is presented in [21]. LEACH-fuzzy logic [22] computes the chance for selecting the CHs. The authors in [23] used fuzzy logic to select heads based on the prediction of residual energy. The authors in [55] have taken node degree and node centrality as fuzzy variables. Initially, each node calculates its cost. In [56], the authors presented a cluster head selection algorithm using ant colony optimization to build load balanced clusters in the network. In [57], the authors presented clustering algorithm using PSO. They considered two types of nodes: normal nodes and high energy nodes. The high energy nodes act as cluster heads in the network, whereas normal sensor nodes act as members of the clusters. Another ant-based clustering (ANTCLUST) method is described in [58]. ANTCLUST protocol categorizes energy-efficient clusters by local interactions among sensor nodes. A hybrid protocol combining Harmony search algorithm (HSA) and PSO is also used for clustering optimization [33]. Honey bee optimization is also used to form clusters in WSNs [34-38]. Wireless sensor network clustering using artificial bee colony algorithm (WSNCABC) [34] uses artificial bee colony to compute the fitness of $\mathrm{CH}$ using the parameters such as residual energy of node and distance from BS to the nodes. However, this algorithm suffers from the high cost for the direct transmission of data from head to the BS. Bee-Sensor$C$ [59] forms clusters and chooses their heads when an event happens. Honey bee mating optimization which motivated the clustering algorthm is presented inBee-C [60]. In [61], route discovery problem is solved after discovering the $\mathrm{CHs}$ using fractional artificial bee colony (FABC) algorithm. The paper presented in [62] is based on the $\mathrm{ABC}$ and the genetic algorithm (GA). CHs are formulated by GA, and their members are decided by ABC. Another bee algorithm-based clustering (BeeWSN) is presented in [63], which creates clusters on the basis of energy of node, degree, speed, and direction.

\section{Bee Colony Model}

The bee colony algorithm is motivated by intelligent foraging behaviour of honey bees. Bee colony has three groups of bees, namely, worker bees, onlooker bees, and scout bees. Probable solution to the optimization problem is represented by the location of a food source, whereas the quality (fitness) of the associated solution corresponds to the nectar amount. Here, the size of the colony is the same as the number of worker bees and also equal to the onlooker bees. The initial locations of food sources are randomly generated, and every worker bee is appointed to a food source. After that, each worker bee finds a new food source in all iteration and computes its quality. Worker bee travels to the new food source if the nectar quantity of the new food source is higher than the earlier one or else it persists with the older one. This process is described by

$$
V_{i j}=x_{i j}+\tau\left(x_{i j}-x_{k j}\right),
$$

where $\tau$ is a random number that lies between $[-1,1], V_{i}$ is new food source, $x_{i}$ is current food source, $x_{k}$ is neighbourhood source, and $j \in\{1,2, \ldots, D\}$ is randomly chosen index with $D$ as dimension of the food source vector. After finishing the search procedure by worker bees, they share the 
information about their food source with onlooker bees. The onlooker bee then assesses the nectar information and picks a food source with a probability related to its nectar amount by

$$
P_{i}=\frac{F_{i}}{\sum_{l=1}^{m} F_{l}},
$$

where $F_{i}$ is the fitness value of the solution $i$ that is proportional to the nectar amount of the food source in the location $i$ and $m$ is the number of food sources.

All onlooker bees find a new neighbouring food source as respective selected food source and analyze its nectar amount. This process continues for a predetermined number of cycles. The food source is allocated as abandoned if any site cannot be improved further. Then, the function transforms; worker bee of that source turns into scout bee. In that location, the scout bee generates a novel solution and is given as

$$
x_{i j}=x_{i j}+\operatorname{rand}(0,1)\left(x_{j \max }-x_{j \min }\right) \text {, }
$$

where abandoned source is represented by $x_{i}$.

\subsection{Proficient Bee Colony-Clustering Protocol (PBC-CP).} As the sensor nodes have restricted energy source, enhancing the network lifetime still remains an important issue. This paper focuses on the need of energy-efficient strategies in wireless sensor network. We propose a proficient bee colony-clustering protocol (PBC-CP) for enhancing the wireless sensor network's lifetime (Algorithm 1). This protocol supports the supervising of a hostile environment wherever replacement of node's battery or even recharging of their battery is not feasible. Generally, the individual nodes' data are often correlated in case of monitoring applications. To send all nodes' data to the BS is not an energy-efficient approach. Therefore, it is beneficial to send aggregated data that illustrate the events taking place in the region. We select clustering infrastructure as the foundation for PBC-CP due to the strongest correlation between statistics from nodes located near to each other. Not all individual nodes send their individual data directly to BS. They send their data to respective $\mathrm{CH}$; then, $\mathrm{CH}$ aggregates the data coming from its member nodes and sends it to the BS. Therefore, only aggregated data are transmitted to BS and not individual node's data. In this way, transmission energy is saved.

Honey bees are highly organized organisms capable of individual cognitive abilities and self-organization. They exhibit a combination of individual traits and social cooperation. We adopt a centralized mechanism for clustering which is managed and controlled at the base station, whereas the routing is performed in a distributed manner. Therefore, the proposed protocol systematically behaves in a semidistributed manner.

Selection of heads of clusters with important parameters is extremely needed to balance the load of the network. A high energy node needs to be selected as $\mathrm{CH}$, as it has to bear extra responsibility of the head. Therefore, we take residual energy into consideration. For minimizing the transfer energy, distance is also taken as important parameter. The number of connecting nodes to the $\mathrm{CH}$ (so-called node degree) should be taken into consideration for uniform load distribution among the heads.

This algorithm works in given distinct phases. We will discuss each of them separately as follows:

(a) Network initialization: initially, the sensor nodes are deployed randomly in the sensing region. The BS transmits beacon signals to all nodes. These beacon signals contain the position information of the base station. Then, all the nodes compute their respective Euclidian distance from the base station. Furthermore, the distance between neighbouring nodes is computed on the basis of arriving strength of signals and their relative coordinates.

(b) Cluster head selection phase: selection of cluster heads depends on fitness function which is computed by bee colony algorithm.

(c) Recruiting cluster members' phase: all the selected heads transmit an information message to the rest of the sensor nodes. This message conveys the information regarding their selection as heads. When the noncluster head nodes get this message, they have to take a decision to be a member under a particular head. This depends on the signal strength of the arrived message. Based on this decision, the non- $\mathrm{CH}$ nodes then report to the appropriate heads to be a member of their cluster. Furthermore, the $\mathrm{CH}$ creates a schedule based on time division multiple access (TDMA) and allocates it to the members of its cluster.

(d) Data gathering: in a cluster, each cluster member transmits its information to their respective heads by the TDMA-based method. We assume it is perfect transmission and no retransmission is required.

(e) Data aggregation: upon receiving the data from all the members, the $\mathrm{CHs}$ aggregate all incoming data together with their own data. In this way, redundancy is reduced if any.

(f) Data transmission: then, cluster heads transmit their aggregated data to the next $\mathrm{CH}$ or $\mathrm{BS}$ in an energyefficient manner. First, $\mathrm{CH}$ checks for the distance between its adjacent $\mathrm{CH}$ and $\mathrm{BS}$. $\mathrm{CH}$ chooses the one which has less distance. If it is BS, then the cluster head transmits its data. But if it is another head, then the sender cluster head checks the residual energy of the adjacent heads and sends its data to the higher one.

(g) Rotation of cluster head: energy of $\mathrm{CH}$ drains out faster because they are involved in carrying out additional tasks such as data gathering from member nodes, aggregation of data, and data relaying, compared to other sensor nodes. Therefore, there is a need to rotate their role. Process of reelection is triggered after each round of data transmission for balancing the energy consumption of all sensor nodes. However, the reelection will be done based on fitness function. 
The fitness function, represented as $f(i)$ is specified as follows:

$$
f(i)=\operatorname{optimize}\left[k\left\{R_{e}(i)+N_{D}\right\}+(1-k)\left\{\frac{1}{E_{u}(i, b)}\right\}\right],
$$

where $k$ is the scaling factor; residual energy of node $\left(R_{e}\right)$ is the ratio of remaining energy to the initial energy in the node; node degree $\left(N_{D}\right)$ is the number of connecting nodes to a particular node within its transmission range; and Euclidean distance from node $i$ to base station is represented by $E_{u}(i b)$.

3.2. Network Model. In this paper, we use the radio propagation model specified in [41] as shown in Figure 2. In a radio model, the signal received at the receiver transmitted from the transmitter with a distance $d$ is given by

$$
P_{r}=\frac{P_{t} G_{t} G_{r} \lambda^{2}}{(4 \pi)^{2} d^{\beta} \text { Loss }}
$$

where $G_{r}$ is the receiver antenna gain, $G_{t}$ is the transmitter antenna gain, $\lambda$ is the carrier wavelength, $\beta$ is the propagation loss factor, and any extra loss in transmitting the packet is represented by Loss.

$$
E_{T}= \begin{cases}l E_{e}+l E_{f s} d^{2}, & \text { if } d<d_{o}, \\ l E_{e}+l E_{t g} d^{4}, & \text { if } d \geq d_{o},\end{cases}
$$

Radio propagation models are free space model and tworay ground propagation model. In the free space propagation model, the propagation loss of transmitting power is inversely proportional to square of the distance between transmitter and receiver. In case of the two-ray ground propagation model, the propagation loss of transmitting power is inversely proportional to fourth power of the distance between transmitter ( $\mathrm{Tx})$ and receiver $(\mathrm{Rx})$. The energy consumption to transmit $l$-bit packet from $\mathrm{Tx}$ to $\mathrm{Rx}$ at the distance $d$ is given bywhere $E_{e}$ is considered as the energy/bit absorbed in the transceiver circuitry and second factor $l E_{f s} d^{2}$ or $l E_{f s} d^{4}$ is considered as the energy/bit absorbed in the power amplifier. The crossover distance $d_{o}$ can be obtained from

$$
d_{o}=\sqrt{\frac{E_{f s}}{E_{t g}}}
$$

If the value of $d_{o}$ is greater than the value of distance between $\mathrm{Tx}$ and $\mathrm{Rx}$, then the free space model is utilized; otherwise, the two-ray ground model is taken into unt. Energy consumption for receiving a $l$-bits message [41] is

$$
E_{R}=l E e .
$$

\section{Experimental Setup}

All experiments were implemented in MATLAB 2009a and run on Windows 7 with Intel (R) Core (TM) 2 Duo T6570 CPU@2.10 GHz. We assume that all sensor nodes have same
Begin

//Initialization//

(1) Base station broadcasts beacon

(2) All nodes calculate Euclidian distance //Cluster head selection phase//

(3) Generate the initial population

(4) Set cycle to 1

(5) Repeat

(6) For each sensor

(7) Launch artificial bee colony algorithm

(8) Select CHs

(9) Cycle $=$ cycle +1

(10) Until Maximum Cycle Number

(11) End For //cluster-member recruitment//

(12) Set $\mathrm{CH}$ cycle to 1

(13) Repeat

(14) For each $\mathrm{CH}$

(15) Set Hop Cycle to 1

(16) Repeat

(17) For each $\mathrm{CH}$ broadcast REQUEST message;

(18) If (non- $\mathrm{CH}$ node neighbours receive REQUEST message)

(19) Then, non-CH node neighbours send ACCEPTED message to $\mathrm{CH}$;

(20) End if

(21) Hop Cycle $=$ Hop Cycle +1

(22) Until Maximum Hop Cycle Number

(23) End For

(24) $\mathrm{CH}$ cycle $=\mathrm{CH}$ cycle +1

(25) Until Maximum $\mathrm{CH}$ cycle Number

(26) End For End

Algorithm 1: Pseudocode of clustering.

initial energy and the capabilities of all nodes such as processing and communicating are similar. They are not equipped with global positioning system, i.e., they do not have capable antennas with moving capabilities. We also assume that base station is fixed and not limited in terms of energy, memory, and computational power. The LEACH protocol is considered as based for comparative analysis of experimental results focusing on clustering centric benefits in the integration with the protocols. The required simulation parameters for various algorithms are shown in $\mathrm{Ta}$ ble $1[20,33-35]$.

4.1. Performance Evaluation. The performance of our model is estimated on residual energy ( $R E)$ of the network, throughput (Thr) of the network, the number of dead nodes $(N N D)$, and the number of alive nodes $(N A N)$ for different rounds of data transfer.

We compare the results of $\mathrm{PBC}-\mathrm{CP}$ with three standard protocols, namely, LEACH [20], PSO [32], and HSA-PSO [33] for alive nodes, dead nodes, residual energy, and throughput in different rounds as shown in Figure 3. It is evident from Figure 3(a) that the performance of PBC-CP in terms of number of alive nodes is better than other 


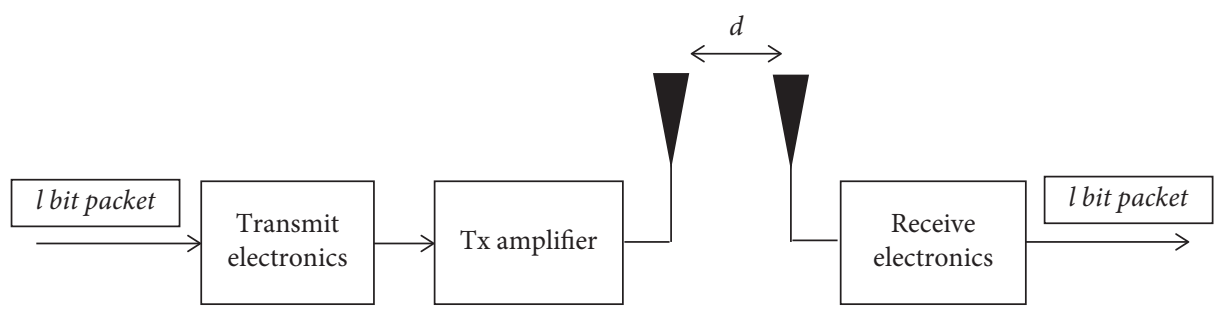

FIgURE 2: Radio hardware model.

TAble 1: Parameters of PBC-CP

\begin{tabular}{lc}
\hline Parameter & Value \\
\hline Sensor field area $(X * Y)(\mathrm{m})$ & $(100 * 200)$ \\
Base station position $(x, y)$ & $(50,150)$ \\
Number of nodes $(\mathrm{s})$ & 100 \\
Initial energy of a node $\left(E_{\mathrm{int}}\right)(\mathrm{J})$ & 0.5 \\
Data packet length $(L)(\mathrm{bits})$ & 4096 \\
Energy/bit absorbed in the transceiver circuitry $E_{e}(\mathrm{~nJ} / \mathrm{bit})$ & 70 \\
Energy/bit absorbed in the power amplifier $\left(E_{f s}\right.$ and $\left.E_{t g}\right)\left(\mathrm{pJ} / \mathrm{bit} / \mathrm{m}^{2}\right)$ & $120 \& 0.0013$ \\
Energy data aggregation $\left(E_{g}\right)(\mathrm{nJ})$ & 5 \\
Number of rounds $\left(R_{\max }\right)$ & 3000 \\
Colony size $(\mathrm{CS})$ & 50 \\
Maximum cycle number $(\mathrm{MCN})$ & 200 \\
Dimension of the food source vector $(\mathrm{D})$ & 20 \\
\hline
\end{tabular}

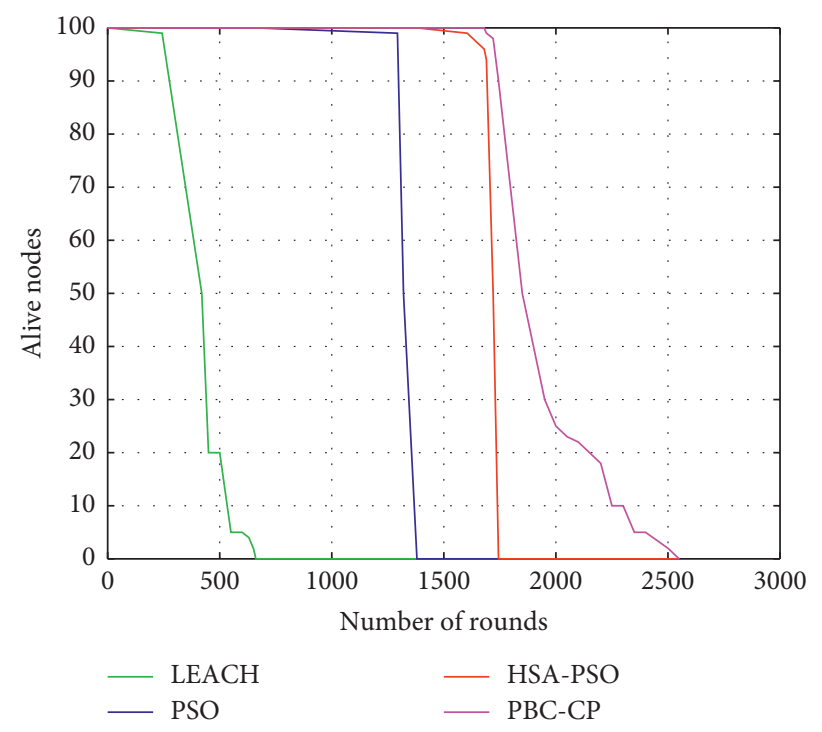

(a)

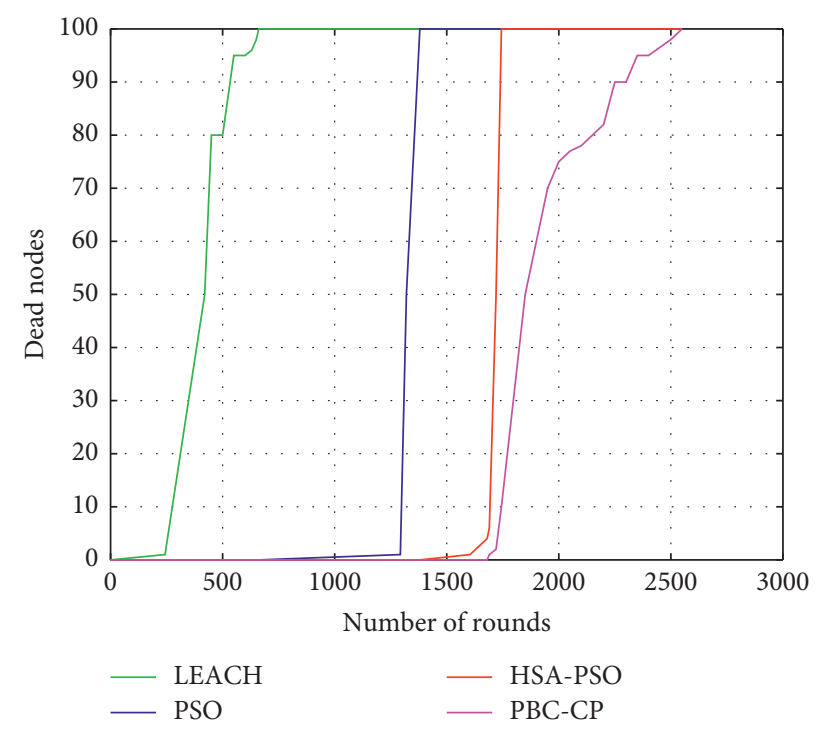

(b)

FIgUre 3: Continued. 


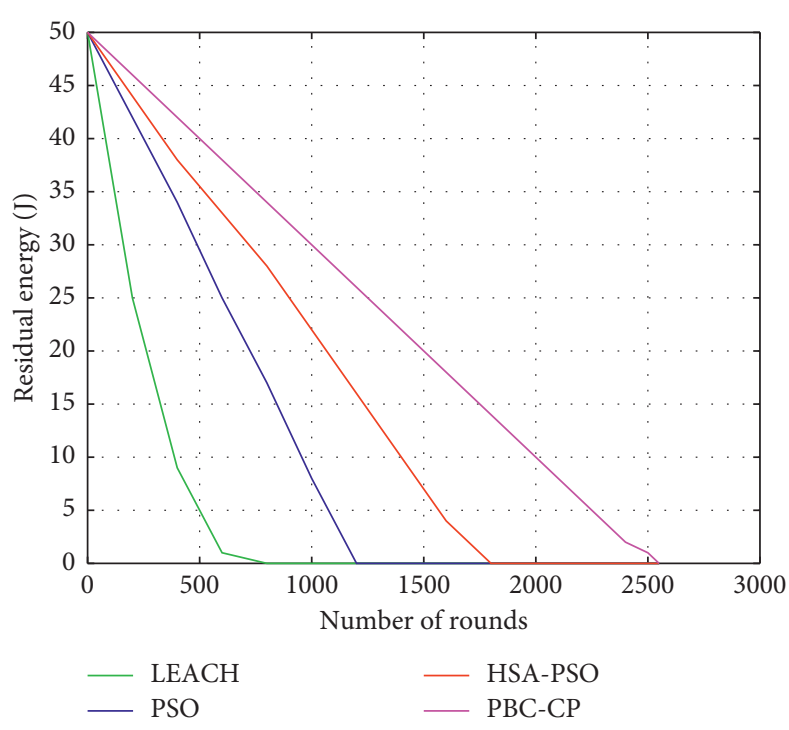

(c)

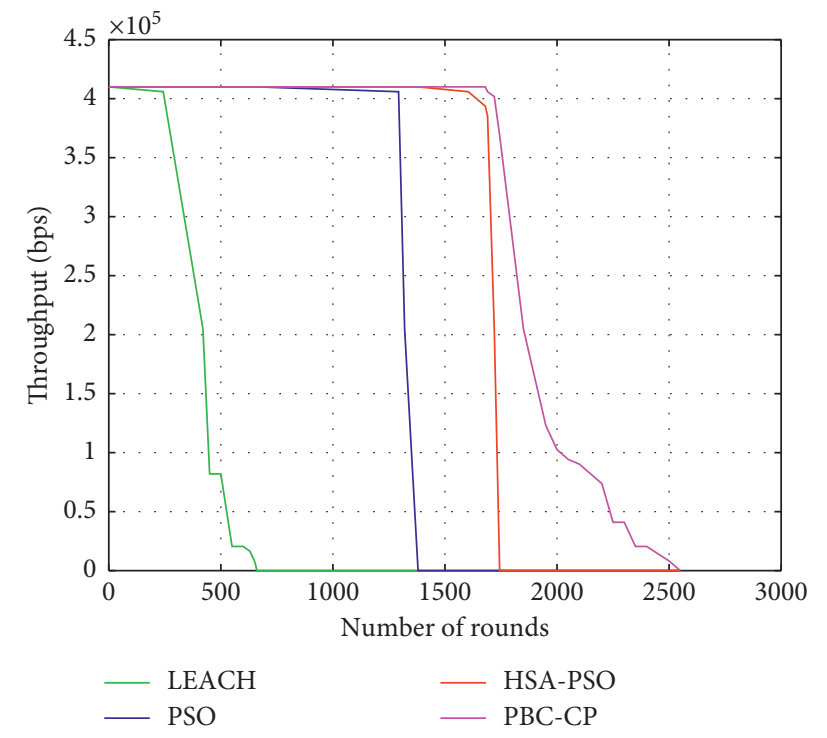

(d)

FIGURE 3: (a) Number of alive nodes vs number of rounds in different protocols. (b) Number of dead nodes vs number of rounds in different protocols. (c) Comparative analysis of $\mathrm{PBC}-\mathrm{CP}$ in terms of residual energy with different rounds. (d) Comparative analysis of $\mathrm{PBC}-\mathrm{CP}$ in terms of throughput with different rounds.

protocols. The alive nodes start dropping in LEACH, PSO, and HSA-PSO after 255 rounds, 1300 rounds, and 1700 rounds, respectively, while alive nodes in PBC-CP start dropping after 1800 rounds. Next, we compare the results of dead nodes in the network for different rounds as shown in Figure 3(b). In LEACH, the first node death occurs near 206 rounds, the reason being probabilistic selection of $\mathrm{CHs}$. HSA-PSO and PSO give improved performance than LEACH. The first dead node in PSO occurs close to 1238 rounds, whereas in HSA-PSO, first node is dead around 1632 rounds. It is now seen from the results that PBC-CP outperforms as the first node is dead here around 1730 rounds.

From Figure 3(c), it is apparent that the PBC-CP demonstrates preeminent results for energy optimization in clustered WSN. The value of $R E$ in LEACH algorithm falls to zero at an early stage (around 650 rounds). It is due to the reason that the heads are selected randomly in the algorithm. PSO algorithm lasts only for around 1400 rounds due to its dynamic capability while HSA-PSO algorithm lasts for almost 1753 rounds due to high exploring efficiency of HSA with the dynamic nature of PSO. In PBC-CP, the value of $R E$ drops to zero after approximately 2500 rounds because of the use of fitness function for the selection of $\mathrm{CHs}$.

The throughput (Thr) of the wireless sensor network for LEACH, PSO, HSA-PSO, and PBC-CP is shown in Figure 3(d). Thr of the network improves as the number of alive node in network enhances. All algorithms have maximum value of $T h r$ equivalent to $0.41 \mathrm{Mbps}$. Nevertheless, when the number of rounds increases, the value of $T h r$ reduces. At 380 rounds, the value of Thr in LEACH is decreased by $52.27 \%$ as compared with the other approaches when value of $T h r$ is equivalent to $0.2 \mathrm{Mbps}$. The value of $T h r$ in PSO is maintained to its maximum value by 1100 rounds, and in HSA-PSO, it is maintained to its maximum value till 1700 rounds. In contrast, the $\mathrm{PBC}-\mathrm{CP}$ algorithm maintains the value of $T h r$ of $0.41 \mathrm{Mbps}$ for more than 2500 rounds.

\section{Conclusion and Future Work}

In this paper, we have presented a proficient bee colonyclustering protocol (PBC-CP) inspired from the foraging principles of honey bees for wireless sensor networks, where the objective is to extend network's lifetime. We select heads of the clusters by exploiting the fast searching features of the bee colony optimization algorithm and transfer data from cluster heads to the base station by energy-efficient path. The simulation results indicate that the $\mathrm{PBC}-\mathrm{CP}$ algorithm outperforms LEACH, PSO, and HSA-PSO. While PBC-CP is an efficient protocol, there may be some more areas for the improvement in this protocol to make it more extensively applicable. In the present implementation of PBC-CP, nodes regularly send their data to their respective heads in their assigned slot. Nodes may only send their data after they notice an event. We have tested PBC-CP in static wireless networks. We may also investigate clustering in mobile sensor networks.

\section{Data Availability}

The data used to support the findings of this study are available from the corresponding author upon request considering the purpose of use in further research in the area.

\section{Conflicts of Interest}

The author declares that there are no conflicts of interest. 


\section{References}

[1] O. Kaiwartya, A. H. Abdullah, Y. Cao et al., "Virtualization in wireless sensor networks: fault tolerant embedding for internet of things," IEEE Internet of Things Journal, vol. 5, no. 2, pp. 571-580, 2018.

[2] A. A. A. Ari, A. Gueroui, N. Labraoui, and B. O. Yenke, "Concepts and evolution of research in the field of wireless sensor networks," International Journal on Computational Networks and Communication, vol. 7, no. 1, pp. 81-98, 2015.

[3] O. Kaiwartya, A. H. Abdullah, Y. Cao et al., "Internet of vehicles: motivation, layered architecture, network model, challenges, and future aspects," IEEE Access, vol. 4, pp. 5356-5373, 2016.

[4] A. Khatri, S. Kumar, O. Kaiwartya, and A. H. Abdullah, "Green computing for wireless sensor networks: optimization and Huffman coding approach," Peer-to-Peer Networking and Applications, vol. 10, no. 3, pp. 592-609, 2017.

[5] L. Farhan, R. Kharel, O. Kaiwartya, M. Hammoudeh, and B. Adebisi, "Towards green computing for Internet of things: energy oriented path and message scheduling approach," Sustainable Cities and Society, vol. 38, pp. 195-204, 2018.

[6] W. Ye, J. Heidemann, and D. Estrin, "Medium access control with coordinated adaptive sleeping for wireless sensor networks," IEEE/ACM Transactions on Networking, vol. 12, no. 3, pp. 493-506, 2004.

[7] A. Khasawneh, M. S. B. A. Latiff, O. Kaiwartya, and H. Chizari, "A reliable energy-efficient pressure-based routing protocol for underwater wireless sensor network," Wireless Networks, vol. 24, no. 6, pp. 2061-2075, 2018.

[8] H. Liu, G. Yao, J. Wu, and L. Shi, "An adaptive energy-efficient and low-latency MAC protocol for wireless sensor networks," Journal of Communications and Networks, vol. 12, no. 5, pp. $510-517,2010$.

[9] A. Khasawneh, M. Latiff, O. Kaiwartya, and H. Chizari, "Next forwarding node selection in underwater wireless sensor networks (UWSNs): techniques and challenges," Information, vol. 8, no. 1, pp. 1-31, 2016.

[10] M. M. Alam, E. Ben Hamida, O. Berder, D. Menard, and O. Sentieys, "A heuristic self-adaptive medium access control for resource-constrained WBAN systems," IEEE Access, vol. 4, pp. 1287-1300, 2016.

[11] R. Willett, A. Martin, and R. Nowak, "Back casting: adaptive sampling for sensor networks," in Proceedings of IEEE International Symposium on Information Processing in Sensor Networks (IPSN), pp. 124-133, Berkeley, CA, USA, April 2004.

[12] L. Tan and M. Wu, "Data reduction in wireless sensor networks: a hierarchical LMS prediction approach," IEEE Sensors Journal, vol. 16, no. 6, pp. 1708-1715, 2016.

[13] N. Wu and S. Latifi, "A survey on data compression in wireless sensor networks," in Proceedings of IEEE International Conference on Information Technology: Coding and Computing, pp. 8-13, Las Vegas, NV, USA, April 2005.

[14] Y. Liu, Q. Zhang, and L. Ni, "Opportunity-based topology control in wireless sensor networks," IEEE Transactions on Parallel and Distributed Systems, vol. 21, no. 3, pp. 405-416, 2010.

[15] R. Zhang and M. A. Labrador, "Energy-aware topology control in heterogeneous wireless multi-hop networks," in Proceedings of IEEE International Symposium on Wireless Pervasive Computing, pp. 1-5, San Juan, PR, USA, February 2007.

[16] O. Kaiwartya, S. Kumar, D. K. Lobiyal, P. K. Tiwari, A. H. Abdullah, and A. N. Hassan, "Multiobjective dynamic vehicle routing problem and time seed based solution using particle swarm optimization," Journal of Sensors, vol. 15, no. 1, pp. pp1-16, 2015.

[17] X. Liu, "A survey on clustering routing protocols in wireless sensor networks," Sensors, vol. 12, no. 8, pp. 11113-11153, 2012.

[18] O. Kaiwartya, S. Kumar, D. Lobiyal, A. Abdullah, and A. Hassan, "Performance improvement in geographic routing for vehicular Ad Hoc networks," Sensors, vol. 14, no. 12, pp. 22342-22371, 2014.

[19] M. M. Afsar and M.-H. Tayarani-N, "Clustering in sensor networks: a literature survey," Journal of Network and Computer Applications, vol. 46, pp. 198-226, 2014.

[20] W. R. Heinzelman, A. Chandrakasan, and H. Balakrishnan, "Energy-efficient communication protocol for wireless microsensor networks," in Proceedings of IEEE International Conference on System Sciences, pp. 10-20, Maui, HI, USA, January 2000.

[21] I. Gupta, D. Riordan, and S. Sampalli, "Cluster-head election using fuzzy logic for wireless sensor networks," in Proceedings of IEEE International Conference on Communication Networks and Services Research (CNSR), pp. 255-260, Halifax, Canada, May 2005.

[22] G. Ran, H. Zhang, and S. Gong, "Improving on leach protocol of wireless sensor networks using fuzzy logic," Journal of Information Computational Science, vol. 7, no. 1, pp. 767-775, 2010.

[23] J.-S. Lee and W.-L. Cheng, "Fuzzy-logic-based clustering approach for wireless sensor networks using energy predication," IEEE Sensors Journal, vol. 12, no. 9, pp. 2891-2897, 2012.

[24] N. Nokhanji, Z. M. Hanapi, S. Subramaniam, and M. A. Mohamed, "An energy aware distributed clustering algorithm using fuzzy logic for wireless sensor networks with non-uniform node distribution," Springer Journal on Wireless Personal Communications, vol. 84, no. 1, pp. 395-419, 2015.

[25] P. Nayak and A. Devulapalli, "A fuzzy logic-based clustering algorithm for WSN to extend the network lifetime," IEEE Sensors Journal, vol. 16, no. 1, pp. 137-144, 2016.

[26] M. Mavrovouniotis, C. Li, and S. Yang, "A survey of swarm intelligence for dynamic optimization: algorithms and Applications," Swarm and Evolutionary Computation, vol. 33, pp. 1-17, 2017.

[27] S. Alam, G. Dobbie, Y. S. Koh, P. Riddle, and S. Ur Rehman, "Research on particle swarm optimization based clustering: a systematic review of literature and techniques," Swarm and Evolutionary Computation, vol. 17, pp. 1-13, 2014.

[28] S. J. Nanda and G. Panda, "A survey on nature inspired metaheuristic algorithms for partitional clustering," Swarm and Evolutionary Computation, vol. 16, pp. 1-18, 2014.

[29] D. Karaboga, B. Gorkemli, C. Ozturk, and N. Karaboga, “A comprehensive survey: artificial bee colony $(\mathrm{ABC})$ algorithm and applications," Artificial Intelligence Review, vol. 42, no. 1, pp. 21-57, 2014.

[30] M. Karaboga, G. A. Di Caro, and M. Farooq, "Swarm intelligence based routing protocol for wireless sensor networks: survey and future directions," Information Sciences, vol. 181, no. 20, pp. 4597-4624, 2011.

[31] J. Wang, J. Cao, B. Li, S. Lee, and R. S. Sherratt, "Bio-inspired ant colony optimization based clustering algorithm with mobile sinks for applications in consumer home automation networks," IEEE Transactions on Consumer Electronics, vol. 61, no. 4, pp. 438-444, 2015.

[32] B. Singh and D. K. Lobiyal, "A novel energy- aware cluster head selection based on PSO for WSN," Springer Journal on 
Human Centric Computing and Information Science, vol. 13, no. 2, pp. 6-18, 2012.

[33] T. Shankar, S. Shanmugavel, and A. Rajesh, "Hybrid HSA and PSO algorithm for energy efficient cluster head selection in wireless sensor networks," Swarm and Evolutionary Computation, vol. 30, pp. 1-10, 2016.

[34] S. Okdem, D. Karaboga, and C. Ozturk, "An application of wireless sensor network routing based on artificial Bee colony algorithm," in Proceedings of IEEE International Conference on Evolutionary Computation, pp. 326-330, New Orleans, LA, USA, June 2011.

[35] D. Karaboga, S. Okdem, and C. Ozturk, "Cluster based wireless sensor network routing using artificial bee colony algorithm," Wireless Networks, vol. 18, no. 7, pp. 847-860, 2012.

[36] R. Kumar and D. Kumar, "Multi-objective fractional artificial bee colony algorithm to energy aware routing protocol in wireless sensor network," Wireless Networks, vol. 22, no. 5, pp. 1461-1474, 2016.

[37] A. A. A. Ari, B. O. Yenke, N. Labraoui, I. Damakoa, and A. Gueroui, "A power efficient cluster-based routing algorithm for wireless sensor networks: honeybees swarm intelligence based approach," Journal of Network and Computer Applications, vol. 69, pp. 77-97, 2016.

[38] C. Ozturk, E. Hancer, and D. Karaboga, "Dynamic clustering with improved binary artificial bee colony algorithm," Applied Soft Computing, vol. 28, pp. 69-80, 2015.

[39] R. Madan and S. Lall, "Distributed algorithms for maximum lifetime routing in wireless sensor networks," IEEE Transactions on Wireless Communications, vol. 5, no. 8, pp. 21852193, 2006.

[40] Li. C. Wang, C. W. Wang, and C. M. Liu, "Optimal number of clusters in dense wireless sensor networks: a cross-layer approach," IEEE Transactions on Vehicular Technology, vol. 58, no. 2, pp. 966-976, 2009.

[41] W. B. Heinzelman, A. P. Chandrakasan, and H. Balakrishnan, "An application-specific protocol architecture for wireless microsensor networks," IEEE Transactions on Wireless Communications, vol. 1, no. 4, pp. 660-670, 2002.

[42] A. Manjeshwar and D. Agrawal, "Teen: a routing protocol for enhanced efficiency in wireless sensor networks," in Proceedings of International IEEE Parallel and Distributed Processing Symposium (IPDPS), pp. 2009-2015, San Francisco, CA, USA, April 2001.

[43] M. J. Handy, M. Haase, and D. Timmermann, "Low energy adaptive clustering hierarchy with deterministic cluster head selection," in Proceedings of IEEE International Conference on Mobile and Wireless Communications Networks (MWCN), pp. 368-372, Stockholm, Sweden, September 2002.

[44] O. Younis and S. Fahmy, "Heed: a hybrid, energy-efficient, distributed clustering approach for ad hoc sensor networks," IEEE Transactions on Mobile Computing, vol. 3, no. 4, pp. 366-379, 2004.

[45] S. Bandyopadhyay and E. Coyle, "An energy efficient hierarchical clustering algorithm for wireless sensor networks," in Proceedings of IEEE International Conference on Communications (INFOCOM), pp. 1713-1723, San Francisco, CA, USA, March 2003.

[46] G. Smaragdakis, I. Matta, and A. Bestavros, "SEP: A Stable Election Protocol for clustered heterogeneous wireless sensor networks," in Proceedings of International Conference on Sensor and Actor Network Protocols and Applications (SANPA), pp. 5-31, Boston, MA, USA, August 2004.

[47] C. Wen and W. Sethares, "Automatic decentralized clustering for wireless sensor networks," EURASIP Journal on Wireless
Communication and Networking, vol. 5, no. 1, pp. 686-697, 2005.

[48] Y.-C. Chen and C.-Y. Wen, "Distributed clustering with directional antennas for wireless sensor networks," IEEE Sensors Journal, vol. 13, no. 6, pp. 2166-2180, 2013.

[49] V. Loscri, G. Morabito, and S. Marano, "A two-level hierarchy for low-energy adaptive clustering hierarchy," in Proceedings of IEEE International Vehicular Technology Conference (VTC), pp. 1809-1813, Dallas, TX, USA, September 2005.

[50] S. H. Kang and T. Nguyen, "Distance based thresholds for cluster head selection in wireless sensor networks," IEEE Communications Letters, vol. 16, no. 9, pp. 1396-1399, 2012.

[51] W. Zhuo, "Energy efficient clustering algorithm based on neighbours for wireless sensor networks," Journal of Shanghai Jiaotong University, vol. 15, no. 2, pp. 150-153, 2011.

[52] L. Ying, H. Qi, and W. Li, "Load-balanced clustering algorithm with distributed self- organization for wireless sensor networks," IEEE Sensor Journal, vol. 13, no. 5, pp. 1498-1506, 2013.

[53] S.-S. Wang and Z.-P. Chen, "LCM: a link-aware clustering mechanism for energy-efficient routing in wireless sensor networks," IEEE Sensors Journal, vol. 13, no. 2, pp. 728-736, 2013.

[54] S. A. Gopi and T. Ponnuchamy, "EE-LEACH: development of energy-efficient LEACH Protocol for data gathering in WSN," EURASIP Journal on Wireless Communications and Networking, vol. 1, no. 1, pp. 1-9, 2015.

[55] H. Taheri, P. Neamatollahi, O. M. Younis, S. Naghibzadeh, and M. H. Yaghmaee, "An energy-aware distributed clustering protocol in wireless sensor networks using fuzzy logic," Ad Hoc Networks, vol. 10, no. 7, pp. 1469-1481, 2012.

[56] C. K. Ho and H. T. Ewe, "A hybrid ant colony optimization approach for constructing load -balanced clusters," in Proceedings of IEEE International Conference on Congress on Evolutionary Computation (CEC 2005), pp. 2010-2017, Edinburgh, UK, September 2005.

[57] M. Azharuddin and P. K. Jana, "Particle swarm optimization for maximizing lifetime of wireless sensor networks," Computers \& Electrical Engineering, vol. 51, pp. 26-42, 2016.

[58] J. Kamimura, N. Wakamiya, and M. Murata, “A distributed clustering method for energy-efficient data gathering in sensor networks," International Journal of Wireless and Mobile Computing, vol. 1, no. 2, pp. 113-120, 2006.

[59] X. Cai, Y. Duan, Y. He, J. Yang, and L. Changle, "Bee-SensorC:anenergy-efficient and scalable multipath routing protocol for wireless sensor networks," International Journal on Distributed Sensor Networks, vol. 11, no. 3, pp. 1-14, 2015.

[60] M. Fathian, B. Amiri, and A. Maroosi, "Application of honeybee mating optimization algorithm on clustering," Applied Mathematics and Computation, vol. 190, no. 2, pp. 1502-1513, 2007.

[61] R. Kumar, D. Kumar, and D. Kumar, "EACO and FABC to multi-path data transmission in wireless sensor networks," IET Communications, vol. 11, no. 4, pp. 522-530, 2017.

[62] M. Asadi Zangeneh and M. Ghazvini, "An energy-based clustering method for WSNs using artificial bee colony and genetic algorithm," in Proceedings of IEEE Conference on Swarm Intelligence and Evolutionary Computation (CSIEC), pp. 35-41, Kerman, Iran, March 2017.

[63] M. Ahmad, A. A. Ikram, I. Wahid, N. Inam, and S. Ali, "A bioinspired clustering scheme in wireless sensor networks: BeeWSN," Procedia Computer Science, vol. 130, pp. 206-213, 2018. 